\title{
Revealing Hanny's Voorwerp: radio observations of IC 2497
}

\author{
G. I. G. Józsa ${ }^{1}$, M. A. Garrett $1,2,3$, T. A. Oosterloo ${ }^{1,4}$, H. Rampadarath ${ }^{2,5}$, Z. Paragi ${ }^{5,6}$, \\ H. van Arkel $^{1}$, C. Lintott ${ }^{7}$, W. C. Keel ${ }^{8}$, K. Schawinski ${ }^{9}$, and E. Edmondson ${ }^{10}$ \\ 1 Netherlands Institute for Radio Astronomy, Postbus 2, 7990 AA Dwingeloo, The Netherlands \\ e-mail: jozsa@astron.nl \\ 2 Leiden Observatory, Univ. Leiden, PO Box 9513, 2300 RA Leiden, The Netherlands \\ 3 Centre for Astrophysics and Supercomputing, Univ. Swinburne, Mail number H39, Swinburne University of Technology, \\ PO Box 218, Hawthorn, Victoria 3122, Australia \\ 4 Kapteyn Astronomical Institute, Univ. Groningen, Postbus 800, 9700 AV Groningen, The Netherlands \\ 5 Joint Institute for VLBI in Europe, Postbus 2, 7990 AA Dwingeloo, The Netherlands \\ 6 MTA Research Group for Physical Geodesy and Geodynamics, PO Box 91, 1521 Budapest, Hungary \\ 7 Univ. Oxford, Dept. Physics, Denys Wilkinson Building, Keble Road, Oxford, OX1 3RH, UK \\ 8 Univ. Alabama, Dept. Physics \& Astronomy, Box 870324, University of Alabama, Tuscaloosa, AL 35487-0324, USA \\ 9 Univ. Yale, Dept. Physics, J.W. Gibbs Laboratory, 260 Whitney Avenue, Yale University, New Haven, CT 06511, USA \\ ${ }^{10}$ Institute of Cosmology and Gravitation, Dennis Sciama Building, Burnaby Road, Portsmouth, PO1 3FX, UK
}

Received 29 April 2009 / Accepted 12 May 2009

\section{ABSTRACT}

\begin{abstract}
We present multi-wavelength radio observations in the direction of the spiral galaxy IC 2497 and the neighbouring emission nebula known as "Hanny's Voorwerp". Our WSRT continuum observations at $1.4 \mathrm{GHz}$ and $4.9 \mathrm{GHz}$ reveal the presence of extended emission at the position of the nebulosity, although the bulk of the emission remains unresolved at the centre of the galaxy. e-VLBI $1.65 \mathrm{GHz}$ observations show that on the milliarcsecond-scale, a faint central compact source is present in IC 2497 with a brightness temperature in excess of $4 \times 10^{5} \mathrm{~K}$. With the WSRT, we detect a large reservoir of neutral hydrogen in the proximity of IC 2497 . One cloud complex with a total mass of $5.6 \times 10^{9} M_{\odot}$ to the South of IC 2497 encompasses Hanny's Voorwerp. Another cloud complex is located at the position of a small galaxy group $\sim 100 \mathrm{kpc}$ to the West of IC 2497 with a mass of $2.9 \times 10^{9} M_{\odot}$. Our data hint at a physical connection between the complexes. We also detect $\mathrm{HI}$ in absorption against the central continuum source of IC 2497 . Our observations strongly support the hypothesis that Hanny's Voorwerp is being ionised by an AGN in the centre of IC 2497. In this scenario, a plasma jet associated with the AGN clears a path through the ISM/IGM in the direction of the nebulosity. The large-scale radio continuum emission possibly originates from the interaction between this jet and the large cloud complex in which Hanny's Voorwerp is embedded. The H I kinematics do not fit regular rotation, thus the cloud complex around IC 2497 is probably of tidal origin. From the H I absorption against the central source, we derive a lower limit of $2.8 \pm 0.4 \times 10^{21}$ atoms $\mathrm{cm}^{-2}$ to the $\mathrm{H} \mathrm{I} \mathrm{column}$ density. However, assuming non-standard conditions for the detected gas, we cannot exclude the possibility that the AGN in the centre of IC 2497 is Compton-thick.
\end{abstract}

Key words. galaxies: active - galaxies: IGM - galaxies: individual: IC 2497

\section{Introduction}

The Dutch school teacher, Hanny van Arkel, discovered what is surely one of the most bizarre objects uncovered via the GalaxyZoo.org morphological census (Lintott et al. 2008), SDSS J094103.80+344334.2. This object, now known as "Hanny's Voorwerp", appears as an irregular cloud located $15-25 \mathrm{kpc}$ in projection from the massive disk galaxy IC 2497 (see grey scale plots in Figs. 1 and 2) and has a redshift matching that of the galaxy $\left(V_{\text {sys }}=15056 \pm 40 \mathrm{~km} \mathrm{~s}^{-1}\right.$, taken from the NASA Extragalactic Database NED) to within $300 \mathrm{~km} \mathrm{~s}^{-1}$ (Lintott et al. 2009). The detected [O III] 25007 emission, dominating the optical appearance of the cloud, is distributed over an area of roughly $15^{\prime \prime} \times 25^{\prime \prime}$, corresponding to $15 \mathrm{kpc} \times 25 \mathrm{kpc}$ at the distance of IC $2497\left(D_{3 \mathrm{~K}}=210 \pm 15 \mathrm{Mpc}\right)$. A WHT spectrum shows strong line emission, with high-ionisation lines (He II, $[\mathrm{Ne} \mathrm{V}]$ ) coextensive with the continuum (Lintott et al. 2009). The quiescent kinematics with line widths of less than $100 \mathrm{~km} \mathrm{~s}^{-1}$ (Lintott et al. 2009) and a global velocity gradient of $\sim 100 \mathrm{~km} \mathrm{~s}^{-1}$ make ionisation from photons probable as the predominant ionisation process, rather than ionisation from shocks. The data do not indicate the presence of any ionising source in the immediate proximity of the nebulosity.

The current leading hypothesis is that Hanny's Voorwerp is being illuminated and heated by an AGN situated at the centre of IC 2497. The phenomenon has been studied in observations of other objects (Morganti et al. 1991; Fosbury et al. 1998; Yoshida et al. 2002; Croft et al. 2006) and the hypothesis is supported by optical observations (Lintott et al. 2009), showing an emission spectrum of the nucleus of IC 2497 comparable to a lowionization nuclear emission-line region (LINER) or the narrowline region of a Seyfert galaxy (see also Morganti et al. 1991, and Fig. 3 therein). The FIRST survey catalogue lists a radio continuum source situated at the central position of IC 2497 (White et al. 1997). Hence, Hanny's Voorwerp appears to be a prime example of AGN feedback processes ionising the surrounding IGM.

However, there is some puzzling evidence against this scenario. A non-detection in a short X-ray observation with the Swift satellite (Lintott et al. 2009) implies that the hypothetical 


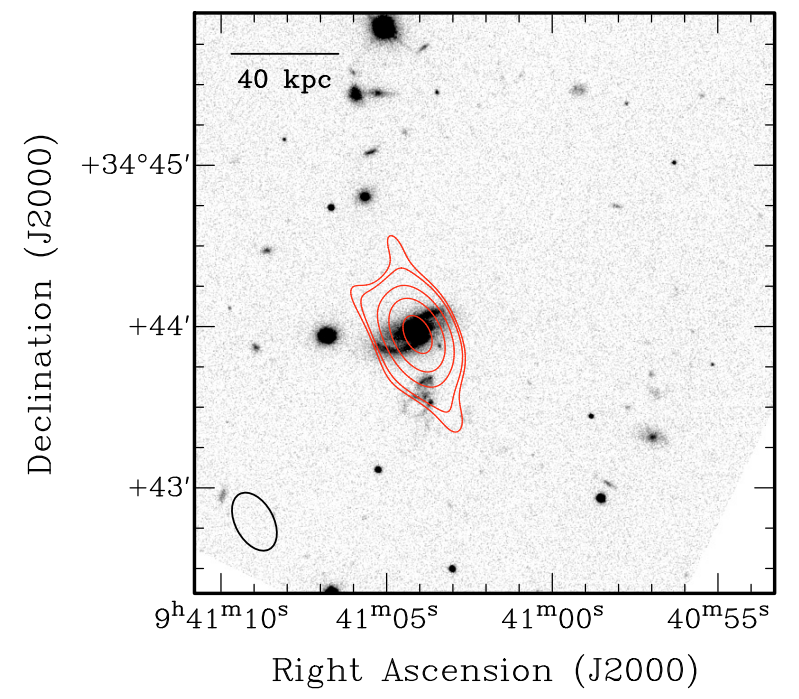

Fig. 1. WSRT 4.9-GHz continuum map (red contours) overlaid on an SDSS $g$-band image of IC 2497. Contours: 0.6, 0.75, 1.5, $3,6 \mathrm{mJy} /$ beam. The ellipse in the lower left corner represents the clean beam HPBW $\left(H P B W=23.3^{\prime \prime} \times 14.2^{\prime \prime}, \sigma_{\text {rms }}=0.15 \mathrm{mJy} / \mathrm{beam}\right)$. To the South of the galaxy, the optical image shows Hanny's Voorwerp.

AGN is Compton-thick towards the observer, but not towards Hanny's Voorwerp. The alternative explanation is that in the short time span of about 80000 years that the radiation needs to reach the nebula, the AGN activity has reduced by a large factor or has even ceased altogether.

The aim of this letter is to present continuum and H I radio observations of IC 2497 and Hanny's Voorwerp, conducted with the Westerbork Synthesis Radio Telescope (WSRT) and by the European VLBI Network (EVN). Our continuum data show extended radio emission at the position of Hanny's Voorwerp. At VLBI resolution, a compact source at the centre of the galaxy is detected. This clearly supports the hypothesis that Hanny's Voorwerp is illuminated and ionised by an AGN hosted by IC 2497. We demonstrate that Hanny's Voorwerp is embedded in a large cloud complex of neutral hydrogen, possibly being the remnant of an interaction of IC 2497 with a galaxy group located at a projected angular distance of 100" from IC 2497.

We describe the observations and the data reduction (Sect. 2), the data (Sect. 3) and discuss our results (Sect. 4).

\section{Observations and data reduction}

Radio continuum observations were performed with the WSRT as a service project in two epochs on 28 September 2008 and 29 September 2008, with a total integration time of $7.5 \mathrm{~h}$. We alternated the receiver frequency between $4.9 \mathrm{GHz}$ and $1.4 \mathrm{GHz}$ with a total bandwidth of $160 \mathrm{MHz}$ to enable an optimally uniform UV coverage in each band. The data underwent a standard data reduction with the Australia Telescope National Facility (ATNF) software package Miriad. The 4.9-GHz map shown in Fig. 1 was generated using visibilities with a baseline length of $<10 \mathrm{k} \lambda$ only and a robust weighting of 0.4 . We found that this was the optimal weighting scheme to map the observed features (see Sect. 3).

In addition, we observed IC 2497 in H I with the WSRT for $2 \times 12 \mathrm{~h}$ on 12 October 2008 and 28 October 2008. We used a total bandwidth of $20 \mathrm{MHz}$, two parallel polarisations, and 1024 channels in total. After a standard data reduction with Miriad we obtained a continuum map, and several data cubes

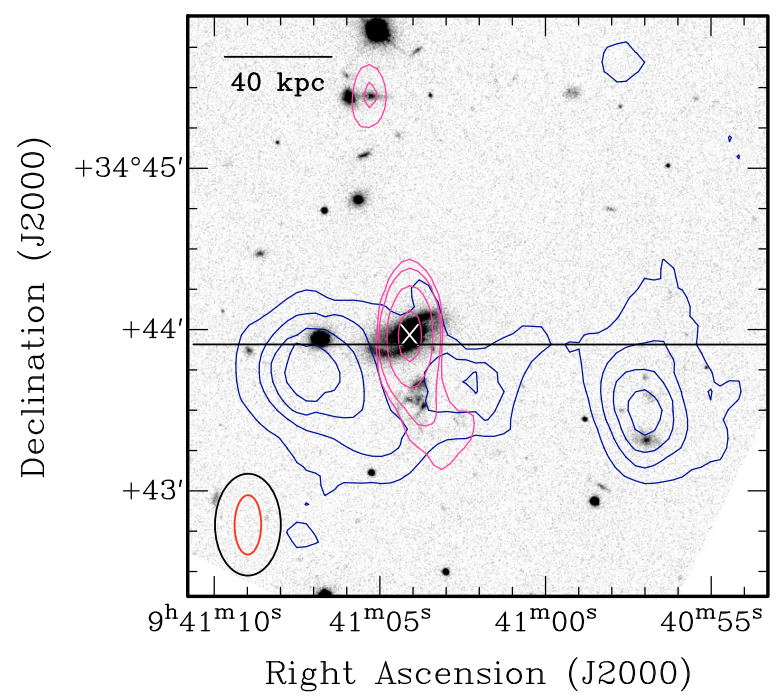

Fig. 2. H I column density (blue contours: $0.5,1,1.5,2 \times$ $10^{20}$ atoms $\mathrm{cm}^{-2}$ ) and $1.4-\mathrm{GHz}$ continuum map (pink contours: 0.35 , $0.7,2.8,11.2 \mathrm{mJy} /$ beam, $\left.\sigma_{\mathrm{rms}}=0.07 \mathrm{mJy} / \mathrm{beam}\right)$ overlaid on the SDSS $g$-band image of IC 2497 (see Fig. 1). The ellipses in the lower left corner represent the clean beam HPBWs, the larger one for the HI measurement $\left(H P B W=37.9^{\prime \prime} \times 24.5^{\prime \prime}\right)$, the smaller one for the continuum image $\left(H P B W=22.1^{\prime \prime} \times 9.9^{\prime \prime}\right)$. The white cross marks the position of the absorption spectrum shown in Fig. 5. The PV diagram shown in Fig. 4 has been taken along the solid line.

each suited for the specific analyses as described below. Due to the better spatial resolution resulting from a complete UV coverage, we use the 1.4-GHz continuum map from the H I observations for our analysis and the one from the dedicated continuum observations as a consistency check. Both maps match within the errors. Figure 2 shows both an integrated H I map, derived from a data cube using natural weighting and a velocity resolution of $108 \mathrm{~km} \mathrm{~s}^{-1}$, and the uniformly weighted $1.4-\mathrm{GHz}$ continuum map.

IC 2497 was observed by the European VLBI network $(\mathrm{EVN})$ in phase reference mode for $2 \mathrm{~h}$ at $1.65 \mathrm{GHz}$ on 30 September 2008. The observing bandwidth was $64 \mathrm{MHz}$ in both LCP and RCP, with 2-bit sampling employed. The array included the Westerbork, Medicina, Onsala 25-m, Torun, Effelsberg, Jodrell Bank MkII, and Darnhall telescopes. The data from each telescope were transported to the correlator at the Joint Institute for VLBI in Europe (JIVE) in real-time, achieving a sustainable data rate of $512 \mathrm{Mbps}$. For the observations, the target was phase-referenced to J0945+3534, a VLBA calibrator located $\sim 1.3$ degrees away from the target source (IC 2497). The data analysis with the National Radio Astronomical Observatory (NRAO) data reduction package AIPS yielded a detection of a single compact source with a signal-to-noise $(S N R)>7$, located at RA $09^{\mathrm{h}} 41^{\mathrm{m}} 04.0875 \pm 0.0002$ and Dec $+34^{\circ} 43^{\prime} 57^{\prime \prime} .778 \pm 0.0^{\prime} 002$ (J2000) with a resolution of 45 milliarcsec $\times 38$ milliarcsec.

\section{Results}

While most of the continuum emission is unresolved in the WSRT observations, positioned at the centre of the galaxy, it is evident from the 1.4-GHz map (Fig. 2) that a faint extension towards the SW is present, stretching out to the location of Hanny's Voorwerp. Also at $4.9 \mathrm{GHz}$ we detect extended radio emission in addition to a dominating point source at the centre of IC 2497 (Fig. 1). 


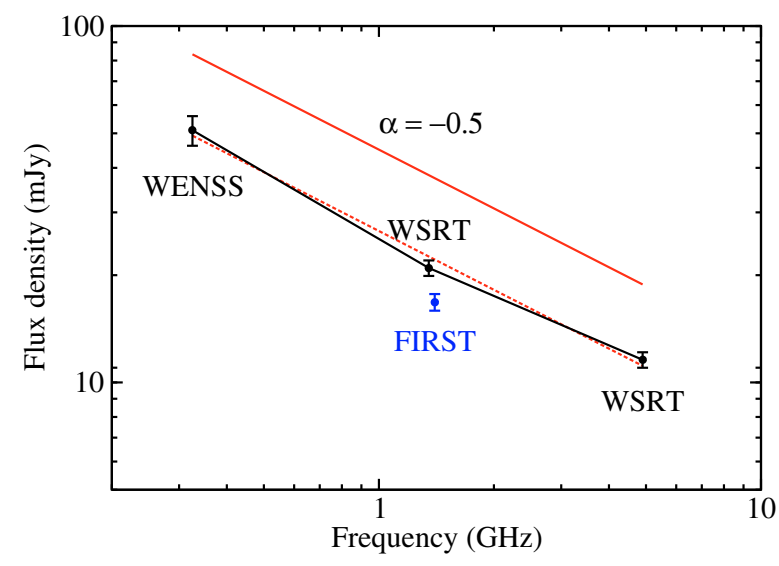

Fig. 3. Continuum flux densities as derived from the WSRT measurements. Also shown is the FIRST flux density at $1.4 \mathrm{GHz}$ and the WENSS result at $0.325 \mathrm{GHz}$. A power-law fit, represented by red lines, yields a spectral index of $\alpha=-0.55$.

At $1.4 \mathrm{GHz}$ we measure a total flux density of $20.9 \pm$ $1.1 \mathrm{mJy}$. This is confirmed in our second, independent continuum map $(21.6 \pm 1.1 \mathrm{mJy})$. The extended part of the emission has a flux density of $3.2 \pm 0.2 \mathrm{mJy}$. The FIRST catalogue (White et al. 1997) reports a flux density of $16.8 \pm 0.9 \mathrm{mJy}$, hence we conclude that probably the extended part of the emission is not detected or resolved out in the FIRST snapshot observation. Taking into account the flux density of $51.0 \pm 4.9$ mJy measured at $325 \mathrm{MHz}$ (Fig. 3) as given in the Westerbork Northern Sky Survey catalogue (WENSS, Rengelink et al. 1997) and the flux densities of $20.9 \pm 1.1 \mathrm{mJy}$ at $1.4 \mathrm{GHz}$ and $11.6 \pm 0.6 \mathrm{mJy}$ at $4.9 \mathrm{GHz}$ derived from our measurements, we fit a power law with a spectral index of $-0.55 \pm 0.05$ (see Fig. 3).

We detect a central, unresolved source in IC 2497 in the WSRT measurements at $1.4 \mathrm{GHz}$ and $4.9 \mathrm{GHz}$. The clear VLBI detection shows the presence of a compact source at the centre of IC 2497 with a flux density of $S_{1.65 \mathrm{GHz}, \mathrm{VLBI}}=1.09 \pm$ $0.14 \mathrm{mJy}$. The observed position (RA $09^{\mathrm{h}} 41^{\mathrm{m}} 04.0875 \pm 0.0002$, Dec $+34^{\circ} 43^{\prime} 57^{\prime \prime}$. $778 \pm 00^{\prime}(002$, J2000) is offset approximately 230 milliarcsec to the Southwest of the VLA FIRST catalogue source at RA $09^{\mathrm{h}} 41^{\mathrm{m}} 04^{\mathrm{s}} .094 \pm 0.023$ and Dec $+34^{\circ} 43^{\prime} 58^{\prime \prime} .00 \pm$ 0.34 (J2000, errors at $90 \%$ confidence level). Both positions are hence identical within the errors. An analysis of the VLBI measurement using the AIPS routine IMFIT suggests a size of $<60$ milliarcsec. The flux density ratio of about $1 / 20$ between the central sources in VLBI- and the WSRT measurements implies that within the WSRT beam the source must be extended on intermediate scales.

We detect H I in the vicinity of IC 2497 at velocities matching the one of IC 2497 with a total mass of about $8.5 \pm 2.1 \times$ $10^{9} M_{\odot}$ (see Fig. 2). Due to the distance of the observed objects, the detections are at a faint level, and we assume an error of 20 percent in the column densities and the total flux. The neutral gas is concentrated in two cloud complexes. One, with a mass of $M_{\mathrm{H} \mathrm{I}, \mathrm{E}}=5.6 \pm 1.4 \times 10^{9} M_{\odot}$, is surrounding the Southern half of IC 2497 with a peak in column density of $2.5 \times 10^{20}$ atoms cm $\mathrm{cm}^{-2}$, roughly located at the position of a small galaxy to the East of IC 2497 . The other has a mass of $M_{\mathrm{H}, \mathrm{W}}=2.9 \pm 0.8 \times 10^{9} M_{\odot}$ and is located to the West at a distance of 94" ( $\approx 96 \mathrm{kpc}$ ) from the centre of IC 2497 , with a peak column density of $2.3 \times$ $10^{20}$ atoms $\mathrm{cm}^{-2}$, located at the position of a small galaxy group. At the position of IC 2497 itself we do not detect a significant

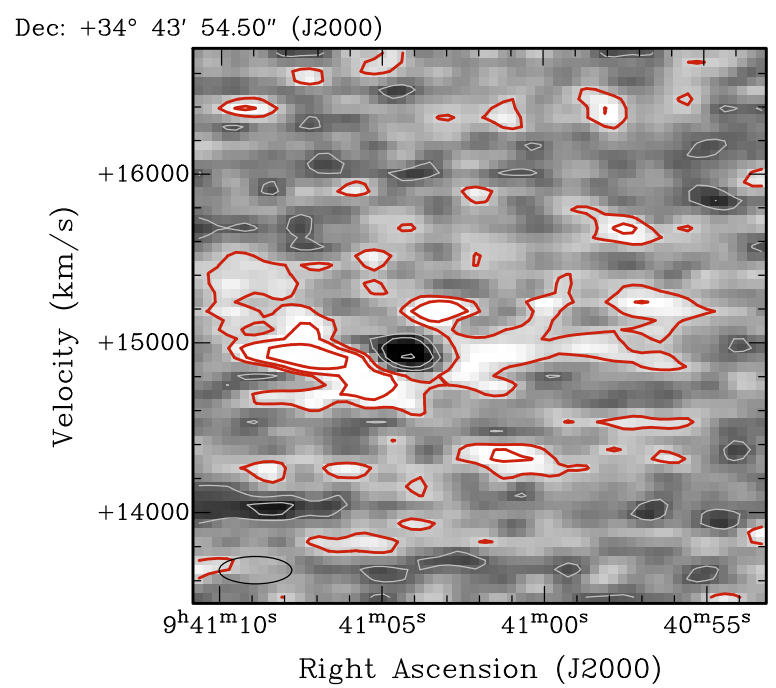

Fig. 4. PV diagram taken along the black line shown in Fig. 2. The Eastern and the Western cloud complexes are connected at a low confidence level also in velocity-space. This indicates a physical connection. The ellipse in the lower left corner indicates the resolution $\left(F W H M=108 \mathrm{~km} \mathrm{~s}^{-1}\right.$, minor axis $\left.H P B W=24.5^{\prime \prime}\right)$. Contours: $-0.6,-0.3,-0.15 \mathrm{mJy} /$ beam (light grey), 0.15, 0.3, 0.6 $\mathrm{mJy} /$ beam (red), $\sigma_{\text {rms }}=0.1 \mathrm{mJy} /$ beam.

amount of $\mathrm{HI}$ in emission. The kinematics of the $\mathrm{HI}$ is complex while showing a gradient towards higher recession velocities from West to East (Fig. 4). Apart from the clear detection shown in Fig. 2, our data hint at the presence of gas spread in the surroundings of IC 2497, albeit at low significance. The most remarkable weak feature is a connection between the Eastern and Western cloud complexes in space and velocity, as indicated in the position-velocity (PV) diagram in Fig. 4. The Eastern cloud complex contains roughly $2 / 3$ of the total detected HI and encompasses the location of Hanny's Voorwerp. While we detect $\mathrm{HI}$ at the position of the extended continuum feature and the nebulosity with a redshift matching that of Hanny's Voorwerp, the column density has a depression at that position.

$\mathrm{HI}$ is also detected in absorption against the central continuum source at the peak position of the continuum. Figure 5 shows the absorption feature as detected in a robust-weighted data cube with a velocity resolution of $72 \mathrm{~km} \mathrm{~s}^{-1}$. The total absorbed Hi flux contained in a beam of $F_{\mathrm{HI}}^{\mathrm{abs}}=0.28 \pm$ $0.03 \mathrm{Jy} \mathrm{km} \mathrm{s}^{-1}$ was determined from the spectrum, using a Gaussian- and second-order polynomial fit for an additional background subtraction. A Gaussian with a width of $F W H M_{\mathrm{HI}}^{\mathrm{abs}}=226 \pm 22 \mathrm{~km} \mathrm{~s}^{-1}$ (corrected for instrumental broadening) represents the absorption profile well (Fig. 5). The H I column density was estimated using the flux density of the unresolved WSRT continuum detection at $1.4 \mathrm{GHz}$, under the assumption that the absorbing sheet covers the background continuum source. We derive a column density of $N_{\mathrm{HI}}^{\mathrm{abs}}=2.8 \pm$ $0.4 \times 10^{21}$ atoms cm $\mathrm{cm}^{-2} \frac{T_{\text {spin }}}{100 \mathrm{~K}} \frac{1}{f}$, where $T_{\text {spin }}$ is the spin temperature and $f$ a covering factor of the absorbing sheet with respect to the background source.

\section{Discussion}

Our observations support the hypothesis that IC 2497 contains an active galactic nucleus with a radio jet emerging in the direction of Hanny's Voorwerp. 


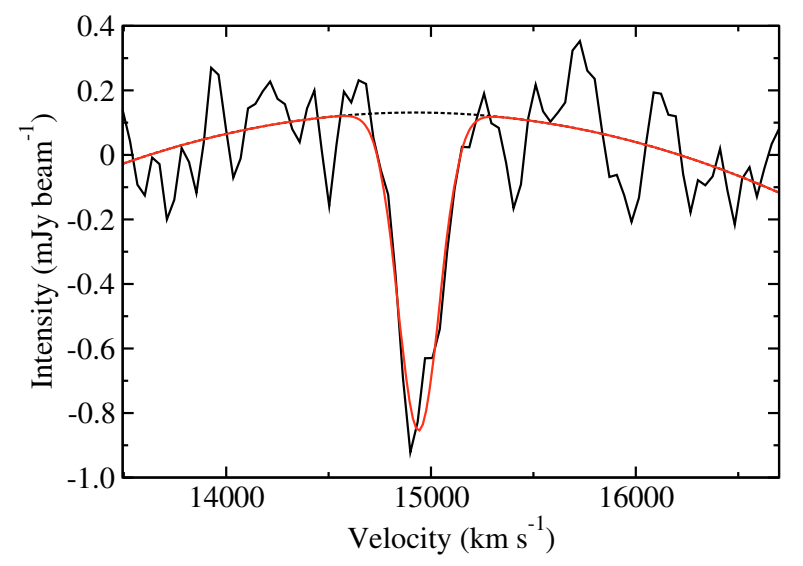

Fig. 5. H I spectrum taken at the position indicated by the white cross in Fig. 2). In black the spectrum is shown, the solid red line is the result of a Gaussian and second-order polynomial fit. The dashed curved line shows the second-order baseline.

From the flux density and the estimated size of the central continuum source detected in our VLBI experiment, we determine a limit on the brightness temperature of the source $T_{\mathrm{b}} \gtrsim 4 \times 10^{5} \mathrm{~K}$. This suggests that the source is probably related to AGN activity in the core of IC 2497. Furthermore, with the WSRT we detect extended continuum emission containing a considerable fraction of the total flux density at $1.4 \mathrm{GHz}$. The extended emission originates at the centre of IC 2497 and points in the direction of Hanny's Voorwerp. The extension is close to perpendicular (at an angle of $76^{\circ}$ to $87^{\circ}$ ) to the optical disk of IC 2497 and reaches a considerable distance from the galaxy (Fig. 2). Most likely, it represents a large-scale radio jet.

The substantial $1.4 \mathrm{GHz}$ radio luminosity $P_{1.4 \mathrm{GHz}}=1.1 \pm$ $0.2 \times 10^{23} \mathrm{~W} \mathrm{~Hz}^{-1}$ and the spectral index $\alpha=-0.55 \pm 0.05$ are consistent with that picture. However, we note that this could also be associated with strong star formation (e.g. Sadler et al. 2002).

The Eastern H I complex has a depression in column density at the position of Hanny's Voorwerp and the extended continuum emission. It seems that the radiation from the central source can reach the gas in Hanny's Voorwerp and ionise it. As a consequence, the density of the neutral gas is lower at the position of the nebulosity. The asymmetric radio jet appears to be visible only in connection to the gas complex detected in $\mathrm{HI}$, where it passes through the IGM, suggesting that it has cleared a path for the radiation from the AGN to reach Hanny's Voorwerp.

Hanny's Voorwerp is thus not an isolated gas cloud. It is embedded in and part of a very large gas complex spanning about $110 \mathrm{kpc}$ in projection. The location of the cloud complex and its irregular morphology and kinematics makes it rather likely that the cloud complex is external in origin. The presence of the Western cloud complex at the location of a galaxy group with rather small members and the tentative kinematical and morphological connection with the Eastern cloud complex suggests a scenario in which both complexes belong to the same structure that contains gas stripped from the galaxy group, instead of gas that has been stripped from a single galaxy. Another candidate as a gas donator or member of the galaxy group is the galaxy located to the East of IC 2497, close to the peak of the total H I intensity. Support for a tidal interaction with a more massive companion rather than a dwarf galaxy is given by the appearance of IC 2497 in the optical. IC 2497 seems to exhibit a massive warp.
The detection of $\mathrm{H} \mathrm{I}$ in absorption already shows that the radiation emerging from the centre of IC 2497 towards us passes through the ISM/IGM at the location of IC 2497. The H I column density of the absorbing material as given above is a lower limit, assuming that the absorbing material is part of the cool, extended ISM. This assumption is supported by extended dust lanes crossing the centre of the galaxy, already visible in the SDSS images, indicating the existence of such an extended component of the ISM (see also Lintott et al. 2009). However, we cannot exclude a scenario in which (part of) the absorption takes place closer to the nucleus, in a compact, circumnuclear disk. This might scale the H I-column density by two factors. Firstly, the covering factor $f$ might differ substantially from 1 . Scaling the H I column density by a factor of up to $1 / f \gtrsim 10$ does not contradict our results, taking into account that we measure a ratio of low- and high resolution flux densities of $\sim 20$. The second factor is the assumed spin temperature of the standard value of $100 \mathrm{~K}$ for $\mathrm{H} \mathrm{I}$ in normal spiral galaxies. Under the assumption of a covering factor of 0.1 , a shielding column density of $\gtrsim 10^{24}$ atoms cm $\mathrm{cm}^{-2}$ would imply a spin temperature of $\gtrsim 3600 \mathrm{~K}$, a realistic value for a gas residing in a circumnuclear disk (Bahcall \& Ekers 1969). Hence, the H I column density might well reach the Comptonthick regime. This would offer an alternative to a short-time scale variability of the AGN as an explanation for non-detections of the AGN at other wavelengths. Sensitive radio observations at high resolution and forthcoming X-ray observations with Suzaku and XMM-Newton will help to solve this issue.

Our observations consistently support the picture that the nebulosity called Hanny's Voorwerp is being illuminated and heated by an AGN situated at the centre of IC 2497. The emission nebula is part of a large cloud complex detected in $\mathrm{HI}$. We detect $\mathrm{HI}$ in absorption against the central continuum source, indicating the possibility that the AGN is Compton-thick and hence not yet detected at other wavelengths. Future observations will help to clarify this.

Acknowledgements. The Westerbork Synthesis Radio Telescope (WSRT) is operated by the ASTRON (Netherlands Foundation for Research in Astronomy) with support from the Netherlands Foundation for Scientific Research (NWO). We thank the director of the Radio Observatory division of ASTRON, Rene Vermeulen, for allocating Director's time for this project, and the WSRT staff for making the observations possible at short notice. The European VLBI Network is a joint facility of European, Chinese, South African and other radio astronomy institutes funded by their national research councils. e-VLBI developments in Europe are supported by the EC DG-INFSO funded Communications Network Development project "EXPReS", Contract No. 02662. We thank the EVN PC chair, Tiziana Venturi, for her approval of the e-EVN observations at short notice. This research was supported by the EU Framework 6 Marie Curie Early Stage Training programme under contract number MEST-CT-2005-19669 "ESTRELA". This research has made use of the NASA/IPAC Extragalactic Database (NED). This work has made use of the data releases of the Sloan Digital Sky Survey (Adelman-McCarthy et al. 2008).

\section{References}

Adelman-McCarthy, J. K., Agüeros, M. A., Allam, S. S., et al. 2008, ApJS, 175, 297

Bahcall, J. N., \& Ekers, R. D. 1969, ApJ, 157, 1055

Croft, S., van Breugel, W., de Vries, W., et al. 2006, ApJ, 647, 1040

Fosbury, R. A. E., Morganti, R., Wilson, W., et al. 1998, MNRAS, 296, 701

Lintott, C. J., Schawinski, K., Slosar, A., et al. 2008, MNRAS, 389, 1179

Lintott, C. J., Schawinski, K., Keel, A., et al. 2009, MNRAS, submitted

Morganti, R., Robinson, A., Fosbury, R. A. E., et al. 1991, MNRAS, 249, 91

Rengelink, R. B., Tang, Y., de Bruyn, A. G., et al. 1997, A\&AS, 124, 259

Sadler, E. M., Jackson, C. A., Cannon, R. D., et al. 2002, MNRAS, 329, 227

White, R. L., Becker, R. H., Helfand, D. J., \& Gregg, M. D. 1997, ApJ, 475, 479

Yoshida, M., Yagi, M., Okamura, S., et al. 2002, ApJ, 567, 118 